\title{
Tonic Tics in Gilles de la Tourette Syndrome
}

\author{
Justyna Kaczyńska ${ }^{1} \quad$ Piotr Janik ${ }^{1}$ \\ ${ }^{1}$ Department of Neurology, Medical University of Warsaw, Warsaw, \\ Poland \\ Neuropediatrics 2021;52:370-376.
}

Address for correspondence Piotr Janik, MD, PhD, Department of Neurology, Medical University of Warsaw, Banacha 1a, 02-097

Warsaw, Poland (e-mail: piotr.janik@wum.edu.pl).

Abstract



Keywords
- Gilles de la Tourette
syndrome
- Tourette disorder
- tonic tics
- dystonic tics
- simple tics
- complex tics

Aim Tonic tics (TTs) are a part of a clinical picture of Gilles de la Tourette syndrome (GTS) and manifest themselves as sustained and isometric contraction of a muscle group devoid of the movement effect or accompanied by only slight visible motion. The aim of this study was to evaluate the prevalence and phenomenology of TTs, and to assess the clinical associations of TTs with tic severity and comorbidities in patients with GTS.

Methods We performed a one-time registration study in a cohort of 241 consecutive outpatients with GTS aged 5 to 50 years ( 188 males, 153 patients under the age of 18 years). All patients were personally interviewed and examined.

Results TTs occurred in $85.2 \%$ of adults and $63.9 \%$ of children and adolescents. Most frequently reported types of TTs were tensing of the abdomen (58.7\%), neck (52.7\%), and upper limbs (50.3\%). Multivariate statistical analysis showed a significant correlation between TTs and the total number of simple tics, total number of complex tics, and age at evaluation. In the group of children and adolescents, an additional significant variable was the duration of GTS. In the group of adults, significant parameters were total number of simple tics, total number of complex tics, peak tic severity ever experienced, premonitory urges, and the presence of dystonic tics.

Conclusion TTs belong to the tic spectrum, common and early symptoms of GTS, are associated with overall a greater number of tics which are more severe, and with more comorbidities.

\section{Introduction}

Gilles de la Tourette syndrome (GTS) is a complex neuropsychiatric condition. The most significant symptoms of the disease are multiple motor and vocal tics. The disease begins in childhood and affects children, adolescents, and adults worldwide. ${ }^{1}$ The diagnostic criteria according to Diagnostic and Statistical Manual of Mental Disorders-5 include the presence of at least two motor and one vocal tics at some time during the illness, although not necessarily concurrently, onset before age of 18 , the tics may wax and wane in frequency but have persisted for more than 1 year since first tic onset; tics that are neither substance-induced nor due to a general medical condition. ${ }^{2}$

Tics are defined as sudden, rapid, recurrent, nonrhythmic motor movements (motor tics), or vocalizations (vocal tics). ${ }^{3}$ Even though this description corresponds well with motor clonic tics, there are some tics which do not fulfill the above criteria and therefore should be distinguished. Longer and slower movement is typical for dystonic tics (DTs); ${ }^{4}$ blocking tics are a presentation of a rapid cessation of muscle activity and voluntary action, for example, walking or speech;, 5 received

March 26, 2020 accepted after revision

October 9, 2020

published online

January 14, 2021
DOI https://doi.org/ 10.1055/s-0040-1722689. ISSN $0174-304 X$

\footnotetext{
(C) 2021. The Author(s).

This is an open access article published by Thieme under the terms of the Creative Commons Attribution-NonDerivative-NonCommercial-License, permitting copying and reproduction so long as the original work is given appropriate credit. Contents may not be used for commercial purposes, or adapted, remixed, transformed or built upon. (https://creativecommons.org/ licenses/by-nc-nd/4.0/) Georg Thieme Verlag KG, Rüdigerstraße 14, 70469 Stuttgart, Germany
} 
cognitive tics are mental acts that have the equivalent among complex vocal tics such as coprolalia, echolalia, or palilalia and are neither visible nor audible; ${ }^{7}$ and finally tonic tics (TTs), which manifest themselves as sustained, isometric contraction of a muscle group devoid of the movement effect or accompanied by only slight visible motion. We observed that TTs occur in different parts of the body and are often associated with features characteristic for typical tics such as premonitory urges (PU), which are momentarily alleviated by performance of a tic giving the feeling of relief. ${ }^{8}$ We therefore hypothesized that TTs have the same characteristics as classic tics and they are a part of the tic spectrum.

Blinking and other clonic tics usually start as a presenting symptom of GTS at the age of 5 to 7 years. ${ }^{8}$ As the disease progresses, various types of tics may appear at different times. The prevalence of different tics may also vary depending on patients' age. ${ }^{9}$ We suspected that TTs may appear only in some patients and wanted to assess whether they are presenting, early or rather late symptom of GTS.

The most commonly used clinician-rated measure of tic severity is the Yale Global Tic Severity Scale (YGTSS), which include the presence of TTs as well. ${ }^{10,11}$ We hypothesized that TTs add significantly to greater tic severity and overall impairment caused by tics.

Some psychiatric disorders are more common in patients with GTS, this applies especially to Attention Deficit Hyperactivity Disorder (ADHD) and obsessive-compulsive disorder (OCD). ${ }^{12}$ It seems that the more comorbid disorders patients have, the more severe tics they develop. Freeman et al compared the group of patients with GTS without comorbidity with patients with at least one additional comorbid diagnosis. They observed that almost twice as many individuals in the comorbid group had severe tics. ${ }^{13}$

In contrast to typical clonic tics, there are also two types of slow and sustained tics: tonic and DTs. ${ }^{5}$ DTs are defined as sustained muscle contractions frequently causing twisting movements, or abnormal, short-lasting, and unfixed postures. ${ }^{4}$ On EMG typical motor clonic tics last less than $100 \mathrm{~ms}$, while the duration of tonic and DTs is over $300 \mathrm{~ms}^{14}$ and according to Jankovic, TTs last even longer than DTs, that is $>500 \mathrm{~ms}^{5}$ As these "nonrapid" tics share some phenomenology, we hypothesized that the occurrence of both tics may be correlated.

The aim of this study was (1) to assess the prevalence, age of onset, and body distribution of TTs; (2) to investigate whether TTs are related to PU and if they are associated with overall tic severity; (3) to ascertain the associations between TTs and psychiatric comorbidities; (4) to assess the correlations between TTs and DTs; and (5) to distinguish between the clinical associations of TTs for patients under the age of 18 years and adults.

\section{Materials and Methods}

\section{Clinical Evaluation}

All the patients were recruited from one single outpatient clinic, and were personally reviewed and evaluated by the same clinician well-experienced in tic disorders (P.J.). Patients were referred to the clinic by general neurologists and psychiatrists due to problematic diagnosis or tics refractory to treatment or sought medical advice on their own because of troublesome tics. The study was designed as a one-time registration study, as patients were registered in the database only once, and no additional clinical data obtained in follow-up visits were included in the analysis.

YGTSS was used to assess the severity of tics within the last week before the clinical evaluation. ${ }^{10}$ To assess the lifetime intensity of tics we defined descriptively the term peak tic severity, which is worst-ever tic severity experienced by the patient and we questioned it actively. Thus, tics at their most severe were determined retrospectively on the basis of an interview with the patient. This definition prevented inappropriate classification, for example, not to classify the disease as mild in adults with mild tics at the time of evaluation but with a history of severe tics during childhood. Peak tic severity was qualified as mild, moderate, or severe. Mild tics were defined as not related to physical or mental discomfort, problems in relations with peers, lower than expected academic achievements, or the need for treatment. Moderate tics generated only small and temporary restrictions in patients' daily lives (e.g., a few days' absence from school, as well as difficulties with homework). Severe tics caused an inability to continue normal daily activities (e.g., repeating grades, losing one's job or physical discomfort), a significant deterioration in the quality of life and the necessity of pharmacological therapy. The total number of simple tics and total number of complex tics were counted for each patient based on a tic symptom checklist included in YGTSS. We collected information about the presence of PU and a feeling of relief following a tic.

The patients were evaluated for the clinical diagnosis of GTS according to the Diagnostic and Statistical Manual of Mental Disorders criteria valid at the time of evaluation (DSM-IV-TR, DSM-5). All patients were systematically interviewed with the aid of semi-structured interview comprised of demographic and clinical data, which was gathered for all the patients. This schedule is based on the Tourette syndrome International database Consortium Data Entry Form, developed by Freeman et $\mathrm{al}^{13}{ }^{13}$ in which study the investigator (P.J.) participated and subsequently used this form in clinical practice. This interview was slightly modified over time and expanded with the questions on different types of tics including TTs (each symptom was scored as either present or absent). The prevalence of the most common comorbid disorders encountered in GTS was evaluated on the basis of the same, mentioned above, and semi-structured clinical interview. Disorders that were listed in this semi-structured interview were $\mathrm{ADHD}, \mathrm{OCD}$, obsessive compulsive behavior $(\mathrm{OCB})$, depression, anxiety disorder (including different forms of anxiety disorders, for instance phobias, panic disorder, generalized anxiety disorder, and separation anxiety disorder), oppositional defiant disorder, conduct disorder, and autism spectrum disorder. If obsessions and compulsions did not cause marked distress and did not significantly interfere with the person's normal functioning, we diagnosed OCB. The list of obsessions and compulsions included in Yale-Brown Obsessive Compulsive Scale (Y-BOCS) were 
used to establish clinical spectrum of OCD/OCB. Each patient was carefully questioned about all the symptoms listed in the DSM to fulfill the diagnostic criteria of the above-mentioned comorbid disorders. The diagnoses of mental disorders that had been made in psychiatric clinics, before our evaluation, were accepted and included into analyses. For the assessment of comorbidities, no validated rating scales for all adults and most patients under the age 18 were used. One-third of children and adolescents, with more complex psychopathology, were assessed with the M.I.N.I. International Neuropsychiatric Interview for Children and Adolescents. Patients with severe psychiatric comorbidities were referred to psychiatrist to confirm the diagnosis. In this case, the psychiatric diagnosis was considered to be definite, and as such taken into consideration in study analyses.

Collection of clinical data from patients with GTS was approved by the ethics committee of the Medical University of Warsaw (KB/2/2007).

\section{Study Participants}

The cohort of GTS cases comprised 241 consecutive patients aged 5 to 50 years (mean age: $16.3 \pm 9.2 ; 188$ males, $78.0 \%$ ). The subjects were evaluated from 2013 to 2019 . One hundred and fifty-three children and adolescents (63.5\%, mean age: $10.4 \pm 3.0$ ) and 88 adults (mean age: $26.6 \pm 7.1$ ) were enrolled. The mean age of tic onset was $6.3 \pm 2.7$ years. Duration of GTS in children and adolescents ranged 1 to 13 years (mean: $5.0 \pm 2.9$ years) and in adults 6 to 39 years (mean: $17.9 \pm 7.3$ years). One hundred and ninety-eight patients $(82.2 \%)$ had, at least, one psychiatric comorbidity, which includes 75 adults (85.2\%) and 123 patients under the age of 18 years (80.4\%). The number of diagnosed mental disorders ranged from 1 to 7 , the median was 2 (IQR: $1-3$ ) in all GTS patients, but 3 (IQR: 2-4) in the group of adults, and 2 (IQR: 1-3) in the group of children and adolescents.

\section{Definition, Differentiation, and Types of Tonic Tics}

To avoid misidentification of symptoms, we explained to patients what the different types of tics are. TTs manifest themselves as isometric muscle contractions that are associated with no or only slight movement, ${ }^{5}$ while DTs consist of slow, twisting movements resembling dystonia. ${ }^{8}$ The duration of muscle contraction during TTs and DTs is longer compared with clonic tics, but unlike DTs, TTs do not lead to any abnormal postures. ${ }^{14}$ For example, in the case of abdominal tics, we qualified them as tonic if they were felt by the patient as tensing of the abdomen or pulling in the stomach, whereas DT of trunk and abdomen was a twisting movement that lead to an abnormal, usually short-lasting and noticeable posture. Another feature in which these two types of tics differ is that DTs are often painful, ${ }^{15}$ while TTs mostly are not. However, to distinguish TTs from DTs in some patients may be challenging, then careful clinical assessment is required. We assessed whether patients had experienced TTs in the past or were experiencing them at evaluation. We considered tics to be currently present if they had appeared in the last 7 days, consistent with the YGTSS. The presence of TTs was determined on the basis of the interview, occurrence during the examination, or video recording provided by the patient. We did not use superficial EMG to assess currently occurring tics, which is a limitation of our study. We also collected information about the anatomical location of these tics. Patients reported the following types of TTs: tensing of the abdomen, neck, upper limb, lower limb, buttocks, or the whole body.

\section{Statistical Methods}

Continuous numerical variables were described by the use of arithmetic means and standard deviation (mean \pm SD), discrete numerical variables were depicted by the use of medians and quartiles $\left(Q_{1}, Q_{3}\right)$, and categorical (nominal or ordinal) variables were presented as absolute numbers with frequencies (percentages). The Shapiro-Wilk W test was used to assess the normality of distributions. Levene's test was performed to assess the homogeneity of variances. Comparisons for two independent groups, considering the numerical traits, encompassed one-way analysis of variance without replication or the Mann-Whitney U test. Contingency tables, depicting and intersecting two categorical variables, were provided with the Chi-square test of independence with Yate's continuity correction when applicable. Two-tailed procedures were fitted. A level of $p<0.05$ was deemed statistically significant. All variables that had been significant factors within the univariate analyses were entered into logistic regression models to determine predictors for TTs in the GTS patients. The regression models were controlled for the studied patients' age and gender. The statistical analyses were performed using Stata/SE release 14.2 (StataCorp LLC, College Station, Texas, United States).

In statistical analysis, we included 232 out of 241 examined patients. Nine patients were excluded as these were young children unable to comprehend questions during the interview, so it was not possible to assess whether they experienced TTs.

\section{Results}

TTs occurred at some point in the lifetime of 167 out of 232 evaluated patients (72\%), including 75 out of 88 adults ( $85.2 \%$ ) and 92 out of 144 children and adolescents (63.9\%). TTs had occurred statistically significantly more often in adults than in children and adolescents $(p<0.001)$. TTs were present in 127 patients (76\%) at the time of evaluation. In 40 patients (24\%), they were present only in the past. The age of onset was known in 109 patients (65.3\%) and the mean age of onset was $10.4 \pm 4.9$ years. TTs occurred on average $4.0 \pm 4.1$ years after tic onset. Most frequently, the onset of TTs was in childhood (67\% at the age of $3-11$ years), less frequent in adolescence (28.4\% at the age of $12-17$ years), exceptionally in adulthood ( $4.6 \%$ at the age $\geq 18$ years). In patients with TTs, their number ranged from 1 to 6 . The median was 2 (IQR: $1-3$ ) overall, but 3 (IQR: $2-4$ ) in the group of adults, and 2 (IQR: $1-3$ ) in the group of patients under the age of 18 years.

In more than half of the patients, tensing of the abdomen, neck, and upper limbs was found. Less frequently, patients reported tensing the lower limbs, whole body, and buttocks (-Fig. 1). 


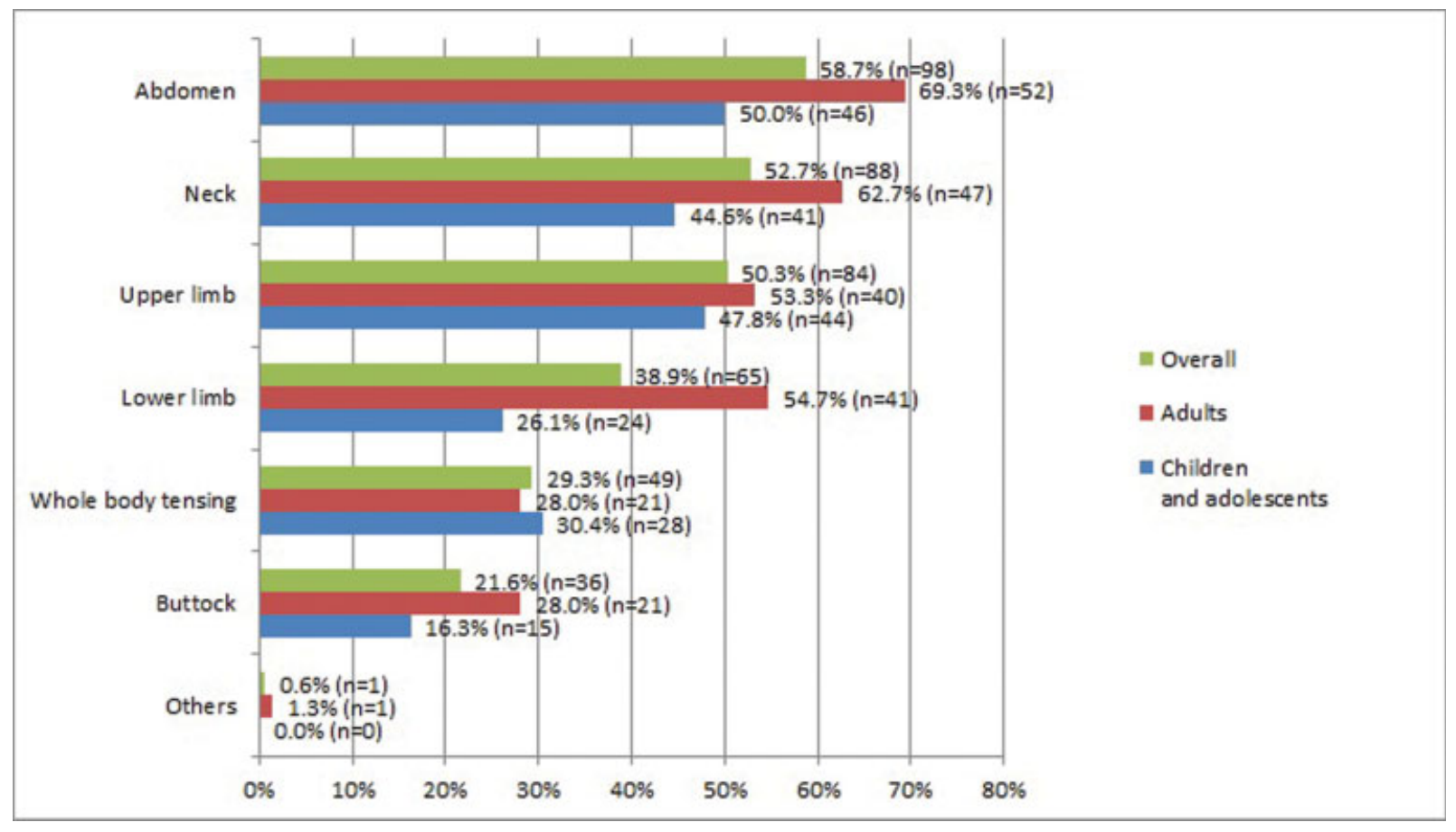

Fig. 1 Prevalence of tonic tics by their location $(n=167)$.

Univariate analysis showed a statistically significant correlation between TTs and peak tic severity, YGTSS, total number of simple tics, total number of complex tics, number of mental disorders, age at the time of evaluation, duration of GTS, DTs, presence of PU, and feeling of relief after tic. Univariate analysis performed in the group of children and adolescents showed a statistically significant association between TTs and total number of simple tics, total number of complex tics, age at the time of evaluation, and duration of GTS, while in the analysis performed in the group of adults, the correlation between TTs and peak tic severity, total number of simple tics, total number of complex tics, DTs, and PU was statistically significant (- Table 1). Multivariate logistic regression analysis showed only significant association between TTs and age at the time of evaluation, total number of simple tics, and total number of complex tics. In the multivariate analysis performed in a group of children and adolescents statistically significant parameters were total number of simple tics, total number of complex tics, age at the time of evaluation, and duration of GTS, while in the analysis for the group of adults, the total number of simple tics, total number of complex tics, peak tic severity, DTs, and PU turned out to be statistically significant ( - Table $\mathbf{2}$ ).

\section{Discussion}

This publication shows that TTs are a common symptom of GTS. In our study group, TTs occurred at some point in the lifetime in 7 out of 10 consecutively examined patients. TTs were reported more often by adult patients, which results probably from longer disease duration and greater age at

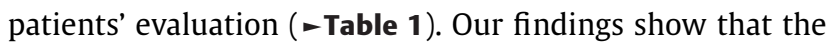
risk of TTs increases with the duration of the disease but only in childhood and adolescence, which suggests that in most patients TTs will appear during these periods. This thesis seems to be confirmed by the fact that the group of nonadult patients with TTs was significantly older than the group of nonadult patients without such tics ( $\mathbf{-}$ Tables $\mathbf{1}$ and $\mathbf{2}$ ). Age at onset of TTs was known in two-third of patients. In nearly $67 \%$ of them, TTs had their onset in childhood, less frequently in adolescence, and onset in adulthood was found exceptionally. We assume that even in the few patients who reported the onset of TTs in adulthood, these tics have actually occurred before. Due to recall bias, many adult patients do not remember the symptoms that occurred in childhood and they usually come alone for outpatient visits, without parents. Mean age of TTs onset was just over 10 years, which was on average 4 years after first tic onset. Thus, TTs seem to be an early symptom of GTS, and although they cannot be considered as a presenting symptom, they seem to join classic tics after only a few years of the disease.

Our study shows that TTs may be present as a single phenomenon or may occur as a few tics in the same patient. In the study group, the number of these tics ranged from 1 to even 6, the median was 2 (IQR: 1-3). Adult patients reported more types of TTs compared with children and adolescents, which probably results from longer disease duration or greater awareness of tic symptoms. Tensing the axial muscles (abdomen and neck) was found most commonly, followed by tensing the limb muscles. TTs occurred more often within the upper part of the body. Tensing the abdomen was the most frequently reported tonic tic among our patients (-Fig. 1). Therefore, the incidence of TTs in different parts of the body differs from that observed for classic tics. Typically, motor tics located most often in the 


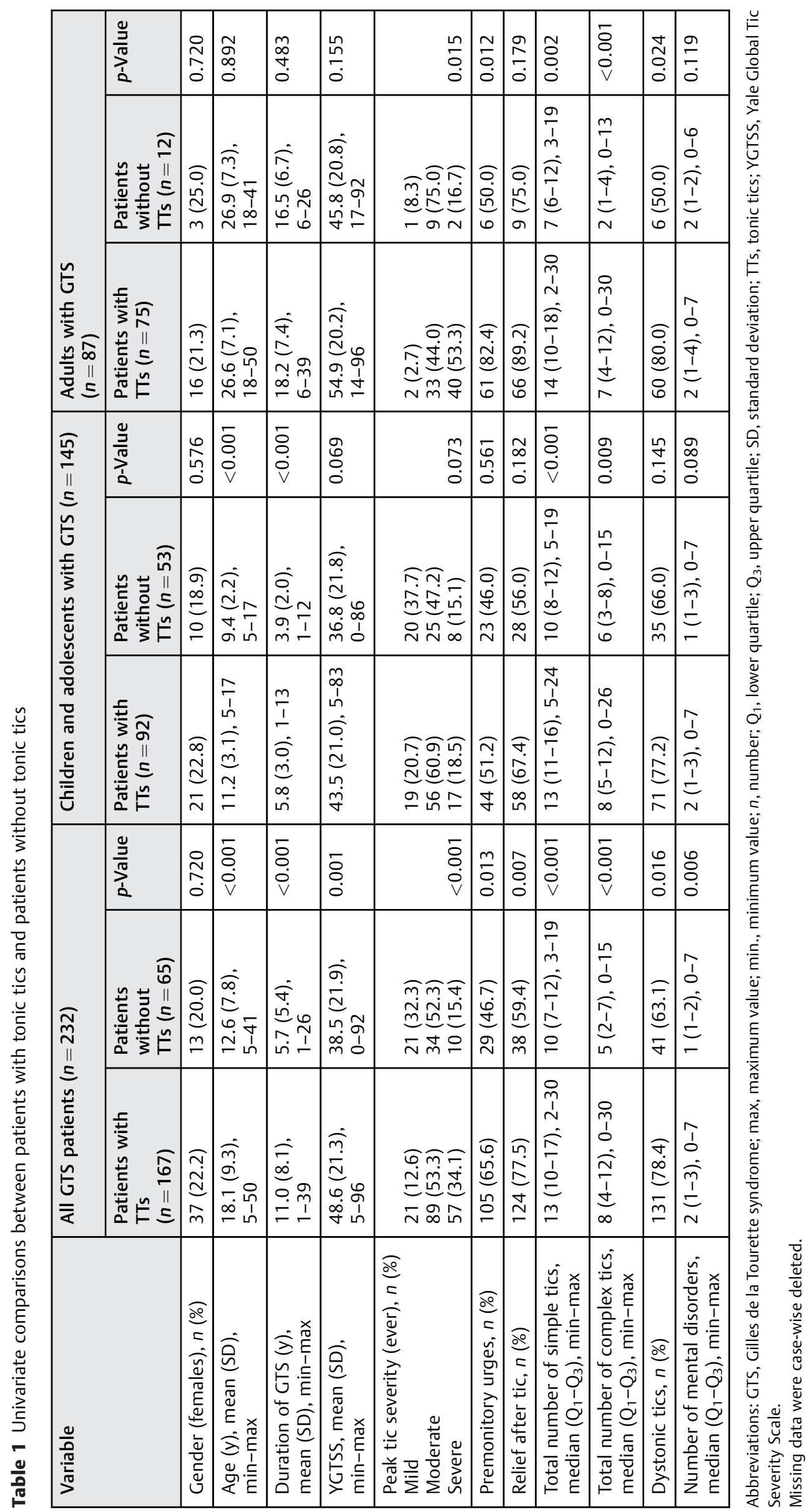


Table 2 Multivariate logistic regression estimates

\begin{tabular}{|c|c|c|c|c|c|c|c|c|c|}
\hline \multirow[t]{2}{*}{ Variable } & \multicolumn{3}{|c|}{ All GTS patients $(n=232)$} & \multicolumn{3}{|c|}{$\begin{array}{l}\text { Children and adolescents } \\
\text { with GTS }(n=145)\end{array}$} & \multicolumn{3}{|c|}{ Adults with GTS $(n=87)$} \\
\hline & OR & $95 \% \mathrm{Cl}$ & $p$-Value & OR & $95 \% \mathrm{Cl}$ & $p$-Value & OR & $95 \% \mathrm{Cl}$ & $p$-Value \\
\hline Gender (females) & 1.64 & $0.69-3.91$ & 0.264 & 1.27 & $0.55-2.95$ & 0.576 & 0.81 & $0.20-3.36$ & 0.776 \\
\hline Age (y) & 1.06 & $1.02-1.12$ & 0.009 & 1.28 & $1.11-1.47$ & $<0.001$ & 0.99 & $0.91-1.08$ & 0.890 \\
\hline Duration of GTS $(y)$ & 0.95 & $0.81-1.11$ & 0.484 & 1.31 & $1.13-1.51$ & $<0.001$ & & & \\
\hline YGTSS & 0.98 & $0.95-1.02$ & 0.336 & & & & & & \\
\hline Peak tic severity & 1.86 & $0.78-4.43$ & 0.164 & & & & 3.80 & $1.27-11.84$ & 0.017 \\
\hline Premonitory urges & 1.03 & $0.34-3.16$ & 0.954 & & & & 4.69 & $1.30-16.88$ & 0.018 \\
\hline Relief after tic & 2.08 & $0.93-4.66$ & 0.076 & & & & & & \\
\hline Total number of simple tics & 1.17 & $1.05-1.31$ & 0.005 & 1.23 & $1.10-1.38$ & $<0.001$ & 1.24 & $1.06-1.46$ & 0.007 \\
\hline Total number of complex tics & 1.11 & $1.02-1.22$ & 0.023 & 1.12 & $1.03-1.23$ & 0.012 & 1.40 & $1.09-1.79$ & 0.008 \\
\hline Dystonic tics & 0.82 & $0.34-1.94$ & 0.647 & & & & 4.00 & $1.13-14.17$ & 0.032 \\
\hline Number of mental disorders & 0.80 & $0.60-1.05$ & 0.105 & & & & & & \\
\hline
\end{tabular}

Abbreviations: Cl, confidence interval; GTS, Gilles de la Tourette syndrome; OR, odds ratio; YGTSS, Yale Global Tic Severity Scale.

eyes, face, and head are a presenting symptom of GTS, followed by the neck and shoulders, later and less often they affect the arms and hands. Motor tics of the trunk and legs are the least common. ${ }^{8,16}$

Occurrence of PU and a feeling of relief following a tic are another characteristic features of tics. ${ }^{1}$ Univariate analysis showed statistically significantly more frequent occurrence of PU in the group of patients with TTs compared with the group without these tics ( $\mathbf{- T a b l e ~} \mathbf{1}$ ). According to the literature approximately $90 \%$ of adults and $37 \%$ of children with GTS report the occurrence of PU. ${ }^{17,18}$ Thus, it is not surprising that TTs were associated with PU more often in adults than in children and adolescents (-Tables $\mathbf{1}$ and $\mathbf{2}$ ). In a study by Leckman et al, 93\% from 135 evaluated patients reported experiencing PU. The mean age at which the patients first became aware of PU was $10.0 \pm 6.2$ years, which was on average $3.1 \pm 5.7$ years after the onset of tics. ${ }^{19}$ This corresponds to the characteristics of patients with TTs in our cohort- the mean age at onset of TTs was $10.4 \pm 4.9$ years, which was $4.0 \pm 4.1$ years after first tic onset. This additionally proves that patients with TTs can potentially experience and report the occurrence of PU. If clinicians are aware that TTs are often associated with the occurrence of PU, it could increase in the number of patients who would be candidates for behavioral therapy because tic-related PU enable to implicate this first-line treatment. In addition, some patients with GTS perceive tics as voluntary movements made automatically to reduce the PU. ${ }^{20}$ Due to the fact that patients with TTs significantly more often experience PU, they are more likely to consider these tics as voluntary movements compared with GTS patients who do not have TTs. Another typical feature of tics is a feeling of relief after tic, which is related to short-term disappearance of PU. Univariate analysis showed that relief after tic occurs significantly more often in the group of patients with TTs compared with the group without these tics (-Table 1 ). In about third-fourth of patients TTs were present at the time of evaluation, and in about one-fourth they occurred only in the past. This proves that TTs have features characteristic for other tics, such as variability over time, and they can occur and vanish similar to typical tics. The above paragraph proves that TTs have many characteristics of classic tics that enables us to include TTs in the tic spectrum.

In the natural course of GTS, tics commonly have their onset at the age of 4 to 6 years, reach their peak severity at the age of 10-12 years, and then their intensity gradually decreases during adolescence and adulthood. ${ }^{21}$ The mean age of onset of TTs in our patients coincides with the period of greatest severity of tics. This may suggest that this type of tics can add significantly to the impairment caused by tics. The results of our analysis suggest that TTs occur in patients with a more severe form of GTS. We proved that TTs correlate with a higher total number of tics, greater tic severity, and additionally with a greater number of psychiatric disorders.

We found that at least one psychiatric disorder occurred in $82.2 \%$ of our patients. Univariate analysis showed significantly more psychiatric disorders in the group of patients with TTs compared with patients without TTs; however, this correlation was not confirmed neither by multivariate analysis nor by analyses conducted separately in the groups of patients under the age of 18 years and adults ( - Tables 1 and 2). It is not clear whether the presence of TTs reflects a more severe GTS phenotype that is associated with more comorbidities, or whether the presence of concomitant psychiatric disorders increases the risk of TTs.

As TTs and DTs belong to "nonrapid" tics, we suspected that characteristics and clinical correlates might be similar for both these tics. Actually, the prevalence and mean age at onset of TTs and DTs were nearly the same (72 and 73.9\%, and 10.4 and 9.9 years, respectively). Additionally, clinical analysis of DTs performed by our team in nearly the same patient cohort showed similar results compared with those 
we obtained during the analysis of TTs. ${ }^{22}$ Among variables included in analyses of both DTs and TTs, almost the same parameters turned out to be statistically significant: severity of tics at the time of evaluation (YGTSS) and in the past (peak tic severity), number of mental disorders, total number of simple, and total number of complex tics. Only age was not a statistically significant parameter in the analysis of DTs, while it was for TTs. In the present study, we showed that DTs occurred more frequently in patients with TTs, and the positive correlation between TTs and DTs in adult patients was confirmed by multivariate analysis ( - Tables 1 and 2). Similarities of clinical phenomenology between these two types of tics and their coexistence in many patients may confirm their affiliation to the "nonrapid" tic spectrum.

Affiliation of TTs to the tic spectrum demonstrated in this publication suggests that their treatment may be the same as that of classic tics. Although there is a relationship between TTs and the overall severity of tics, the treatment should only be initiated in patients for whom tics are bothersome, as in the case of typical clonic tics. Despite many similarities between TTs and DTs we have described, it seems that the injections with botulinum toxin, although effective in many patients with DTs, ${ }^{15}$ will not find application in patients with TTs. This is due to the fact that TTs affect large muscle groups, for example, the entire limb or the entire abdomen, so it is not possible to administer botulinum toxin to all muscles responsible for causing symptoms.

\section{Conclusion}

The diagnosis of GTS is based on the clinical picture. Therefore, clinicians should carefully search for any tic symptoms and actively ask patients about their occurrence. We showed that TTs belong to the tic spectrum, are common and early symptoms of GTS, and should be differentiated from DTs. We also found that patients with TTs have a greater total number of tics which are more severe, and they have more comorbidities.

Conflict of Interest

None declared.

\section{References}

1 Singer HS. Tics and Tourette syndrome. Continuum (Minneap Minn) 2019;25(04):936-958

2 American Psychiatric Association. Diagnostic and Statistical Manual of Mental Disorders (DSM-5). 5th ed. Washington: American Psychiatric Association; 2013
3 Martino D, Madhusudan N, Zis P, Cavanna AE. An introduction to the clinical phenomenology of Tourette syndrome. Int Rev Neurobiol 2013;112:1-33

4 Jankovic J, Stone L. Dystonic tics in patients with Tourette's syndrome. Mov Disord 1991;6(03):248-252

5 Jankovic J. Tourette syndrome. Phenomenology and classification of tics. Neurol Clin 1997;15(02):267-275

6 Rizzo G, Martino D, Logroscino G. I need to freeze my gait. Mov Disord Clin Pract (Hoboken) 2015;2(04):440-441

7 O'Connor K. Cognitive-Behavioral Management of Tic Disorders. Wiley Online Library. 2005

8 Martino D, Leckman JF. Tourette Syndrome. Oxford University Press; 2013

9 Freeman RD, Zinner SH, Müller-Vahl KR, et al. Coprophenomena in Tourette syndrome. Dev Med Child Neurol 2009;51(03): 218-227

10 Leckman JF, Riddle MA, Hardin MT, et al. The Yale Global Tic Severity Scale: initial testing of a clinician-rated scale of tic severity. J Am Acad Child Adolesc Psychiatry 1989;28(04):566-573

11 McGuire JF, Piacentini J, Storch EA, et al. A multicenter examination and strategic revisions of the Yale Global Tic Severity Scale. Neurology 2018;90(19):e1711-e1719

12 Hirschtritt ME, Lee PC, Pauls DL, et al;Tourette Syndrome Association International Consortium for Genetics. Lifetime prevalence, age of risk, and genetic relationships of comorbid psychiatric disorders in Tourette syndrome. JAMA Psychiatry 2015;72(04): 325-333

13 Freeman RD, Fast DK, Burd L, Kerbeshian J, Robertson MM, Sandor P. An international perspective on Tourette syndrome: selected findings from 3,500 individuals in 22 countries. Dev Med Child Neurol 2000;42(07):436-447

14 Cath DC, Hedderly T, Ludolph AG, et al;ESSTS Guidelines Group. European clinical guidelines for Tourette syndrome and other tic disorders. Part I: assessment. Eur Child Adolesc Psychiatry 2011; 20(04):155-171

15 Jankovic J. Botulinum toxin in the treatment of dystonic tics. Mov Disord 1994;9(03):347-349

16 Jagger J, Prusoff BA, Cohen DJ, Kidd KK, Carbonari CM, John K. The epidemiology of Tourette's syndrome: a pilot study. Schizophr Bull 1982;8(02):267-278

17 Kwak C, Dat Vuong K, Jankovic J. Premonitory sensory phenomenon in Tourette's syndrome. Mov Disord 2003;18(12):1530-1533

18 Banaschewski T, Woerner W, Rothenberger A. Premonitory sensory phenomena and suppressibility of tics in Tourette syndrome: developmental aspects in children and adolescents. Dev Med Child Neurol 2003;45(10):700-703

19 Leckman JF, Walker DE, Cohen DJ. Premonitory urges in Tourette's syndrome. Am J Psychiatry 1993;150(01):98-102

20 Hallett M. Tourette syndrome: update. Brain Dev 2015;37(07): 651-655

21 Robertson MM, Eapen V, Singer HS, et al. Gilles de la Tourette syndrome. Nat Rev Dis Primers 2017;3:16097

22 Szejko N, Jakubczyk A, Dunalska A, Janik P. Dystonic tics in patients with Gilles de la Tourette syndrome. Neurol Neurochir Pol 2019;53(05):335-340 